\title{
Desenvolvimento de uma formulação de argamassa refratária com utilização de resíduos industriais para aplicação na construção civil
}

\author{
Douglas Luiz Giordani ${ }^{1}$ \\ Alexandre Zaccaron ${ }^{1 *}$ (1) \\ Thuanni Gesser Müller ${ }^{2}$ \\ Camila Machado de Oliveira ${ }^{2}$ \\ Fernanda Cristina Restelatto ${ }^{3}$ \\ Michael Peterson 1,2,3
}

\section{Resumo}

A construção civil vem se destacando no Brasil nos últimos anos e as construtoras tiveram que se adequar às necessidades de seus clientes. No sul do Brasil, devido ao tipo de colonização e também devido à cultura das pessoas, há a necessidade das construtoras projetarem prédios residenciais com churrasqueiras, que podem ser a gás ou a carvão. Estas unidades possuem um sistema construtivo adequado para resistir a altas temperaturas e tanto os tijolos quanto a argamassa necessitam ter este tipo de resistência. $O$ trabalho em questão teve como objetivo testar a substituição do cimento por materiais com características pozolânicas, como a cinza de termoelétrica e a cinza de indústria cerâmica na fabricação de argamassa refratária. As misturas formuladas foram avaliadas por dilatometria e resistência ao arrancamento, indicando valores satisfatórios de resistência a altas temperaturas e diminuição da resistência de aderência à tração.

Palavras-chave: Argamassa refratária; Cimento; Valorização de resíduos; Dilatometria.

\section{Development of a refractory mortar formulation using industrial waste for application in civil construction}

\begin{abstract}
The civil construction industry has been highlighting in Brazil in recent years and the construction companies had to adapt to the needs of their customers. In southern Brazil, due to the type of colonization and also due to the culture of the people, there is a need for developers to design residential buildings with barbecues, which can be gas or charcoal. These units have a suitable construction system to withstand high temperatures and both bricks and mortar need to have this type of resistance. This work aimed to test the replacement of cement by materials with pozzolanic characteristics, such as thermoelectric ash and ceramic industry ash in the manufacture of refractory mortar. The formulated mixtures were evaluated by dilatometry and pullout strength, indicating good resistance to high temperatures and decreased tensile strength.
\end{abstract}

Keywords: Refractory mortar; Cement; Waste recovery; Dilatometry.

\section{Introdução}

O cimento é a principal matéria-prima utilizada na construção civil. De acordo com o SNIC [1], no ano de 2016 foram produzidas 57 milhões de toneladas de cimento liberando aproximadamente 35 milhões de toneladas de gás carbônico $\left(\mathrm{CO}_{2}\right)$ na atmosfera.
Devido a este agravante e em vista da necessidade da valorização dos variados tipos de resíduos gerados pelas indústrias, é necessário que se busquem alternativas para a substituição do cimento por estes resíduos para que se minimizem os impactos ao meio ambiente. Com base nisso, a utilização de resíduos

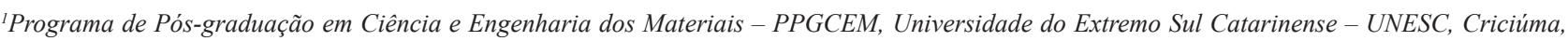
SC, Brasil.

${ }^{2}$ Laboratório de Valoração de Resíduos - LabValora, Universidade do Extremo Sul Catarinense - UNESC, Criciúma, SC, Brasil.

${ }^{3}$ Laboratório de Reatores e Processos Industriais - LabRepi, Universidade do Extremo Sul Catarinense - UNESC, Criciúma, SC, Brasil.

*Autor correspondente: alexandrezaccaron@hotmail.com
} 
como materiais pozolânicos em substituição do cimento tem-se mostrado muito atraente no meio tecnológico [2].

As mais diversas atividades que possuem biomassa (ou outros materiais) como insumo energético para geração de energia, geram cinzas como resíduo [3,4]. Essas cinzas podem ser oriundas das mais diversas fontes, como: cana-deaçúcar [5-7], casca de arroz [8-10], lenha de eucalipto [11-13], termoelétrica a carvão [14-16] entre outros [17-21].

O uso dessas cinzas vem sido amplamente estudados no meio científico, sendo incorporados em cerâmica vermelha [22,23], tijolos de solo cimento [24,25], blocos e artefatos de cimento [26,27] e como argamassa [28-31].

Dentre os resíduos que podem ser incorporados e com potencial gerador, avistou-se a cinza oriunda da queima de fornos cerâmicos, no qual, apresenta um grande problema devido ao seu descarte de forma incorreta [32], e também, as cinzas oriunda das termoelétricas, que já são em suma, materiais usuais na produção de cimento [33].

As argamassas de revestimento utilizadas pela construção civil, podem ser definidas como a mistura homogênea de agregado(s) miúdo(s), aglomerante(s) inorgânico(s) e água, contendo ou não aditivos ou adições, com propriedades de aderência e endurecimento, para os quais vários estudos já foram realizados [34]. Como exemplos, o estudo de Cincotto [35] sobre as principais manifestações patológicas das argamassas de revestimento.

A aderência da argamassa é uma das propriedades de maior importância, considerando as ligações entre os materiais, e foi definida como a capacidade que a interface componente-argamassa possui de absorver tensões tangenciais (cisalhamento) e normais (tração) a ela, sem romper-se [36]. Outro fator a se considerar, é a escassez de mão-de-obra especializada (qualificada) na indústria da construção civil, o que cada vez mais, diminui a qualidade dos resultados. Tal fato onera a produtividade no canteiro de obra [37].

Devido a esses fatores, necessitam-se cada vez mais introduzir produtos que aprimorem a produtividade, com fácil aplicabilidade. Com base nisso, esse trabalho objetivou-se em desenvolver uma argamassa refratária que esteja pronta para o uso, otimizando o tempo em relação a massas tradicionais, que precisam de preparo através da mistura de vários produtos.

\section{Procedimento experimental}

Para realização do estudo, foram utilizados uma argila refratária e cimento Portland CP-IV, cinza proveniente de termoelétrica (Cinza $\mathrm{T}$ ) e cinza proveniente de indústria cerâmica (Cinza P). Para poder ter mais segurança e controle das formulações, fez-se necessário realizar alguns ensaios e caracterização das matérias primas a serem utilizadas.

A análise química da argila refratária e dos resíduos de cinza (Cinza T e Cinza P) foram realizadas por fluorescência de raios-X por meio de um espectrômetro modelo Shimadzu XRD 6000.
A determinação das fases cristalinas das cinzas foi realizada por difratrometria de Raios-X, através de um difratômetro Lab X, modelo XRD 6000, operando a $25 \mathrm{kV}$ e $25 \mathrm{~mA}$, com radiação de $\mathrm{CuK} \alpha(\lambda=1,5406 \AA)$, ângulo de varredura de $3^{\circ}$ a $80^{\circ} 2 \theta$, passo de 0,02 e tempo de contagem de $0,6 \mathrm{~s}$ por passo, ou seja, $2 \% \mathrm{~min}$.

A análise termogravimétrica (TG) e a análise termodiferencial (ATD) foram realizadas nas cinzas em um equipamento marca Netzsch, modelo Jupter. As amostras foram ensaiadas em atmosfera ar, com incremento de $10^{\circ} \mathrm{C} / \mathrm{min}$ até uma temperatura de $1300^{\circ} \mathrm{C}$.

A distribuição do tamanho de partículas das cinzas foi realizada em difrator, modelo Cilas 1064 da marca Acil Weber, através de medidor de partículas a laser em via úmida, com faixa analítica de 0,04 a $500 \mu \mathrm{m}$.

Também foi realizado o ensaio de atividade pozolânica à cal. Regulamentado pela NBR 5751 [38], neste ensaio foram confeccionados 4 corpos de prova cilíndricos para cada resíduo com uma mistura de hidróxido de cálcio, areia, água e a cinza. Após 7 dias os corpos de prova foram ensaiados para a sua resistência à compressão em máquina EMIC de ensaios mecânicos segundo NBR 5739 [39].

Foi utilizado o método BET (Brunauer-Emmet-Teller, marca Quantachrome) para análise da superfície específica dos resíduos.

Utilizou-se como fórmula padrão a mistura comumente utilizada na indústria da construção civil, com uma argila refratária (composta basicamente por 20\% de argila fundente, $70 \%$ argila barro-branco e $10 \%$ chamote) e do cimento Portland CP-IV (Votorantim ${ }^{\circledR}$ ). A proporção recomendada é de:

- $\quad 80 \%$ Argila Refratária;

- $\quad 20 \%$ Cimento Portland CP-IV;

- Água até atingir consistência de argamassa (podendo variar de acordo com a umidade em que se encontra a argila refratária).

Conforme mostra na Tabela 1 , foram desenvolvidas quatro formulações diferentes com os índices de substituição do cimento Portland pelas cinzas variando entre 5 e $10 \%$. Ao todo foram confeccionados 20 corpos-de-prova para cada formulação, dos quais passaram pelos testes tecnológicos.

Posteriormente à análise dos resultados de caracterização das matérias-primas e escolha das melhores composições, as argamassadas desenvolvidas foram ensaiadas quanto à

Tabela 1. Tabela de formulações para a argamassa refratária

\begin{tabular}{ccccc}
\hline Formulações & $\begin{array}{c}\text { Argila } \\
\text { refratária }\end{array}$ & $\begin{array}{c}\text { Cimento } \\
\text { CP-IV }\end{array}$ & Cinza P & Cinza T \\
\hline P5 & 80 & 15 & 5 & - \\
P10 & 80 & 10 & 10 & - \\
T5 & 80 & 15 & - & 5 \\
T10 & 80 & 10 & - & 10 \\
C & 80 & 20 & - & - \\
\hline
\end{tabular}


sua retração linear, análise dilatométrica e resistência ao arrancamento.

A análise dilatométrica, foi realizado em um dilatômetro (NETZSCH modelo DIL 402C).

O ensaio de resistência ao arrancamento ou ensaio de aderência à tração, regido pela NBR 13528 [40] foi realizado com aparelho da marca Dinateste, modelo DNTT-5. Para a sua realização, o equipamento é fixado à superfície da parede refratária e calculado a tensão de tração necessária para romper o substrato de argamassa.

Na Figura 1 estão apresentadas as paredes confeccionas com as formulações de argamassa refratária e tijolos refratários e os corpos de prova durante e após o ensaio de arrancamento.

\section{Resultados e discussão}

Os resultados da análise química da cinza de indústria cerâmica (Cinza P), da cinza de termoelétrica (Cinza T) e da argila refratária são apresentados da Tabela 2.

Conforme NBR 12653 [41], a Cinza P é classificada na classe $\mathrm{E}$, e a Cinza $\mathrm{T}$ na classe $\mathrm{C}$. Desta forma, conforme Tabela 2, devem apresentar somatório de óxidos $\mathrm{SiO}_{2}$, $\mathrm{Al}_{2} \mathrm{O}_{3}, \mathrm{Fe}_{2} \mathrm{O}_{3}$ maior que 50 e $70 \%$. Em ambos os casos este requisito é atendido.

Em relação à perda ao fogo, a porcentagem em massa deveria ser menor que $6 \%$ para ambas as cinzas, requisito que também foi alcançado.

Nota-se que o somatório dos óxidos da argila refratária não atinge $100 \%$, esse fator pode ocorrer devido à presença de enxofre na amostra [42].

Conforme mostra a Figura 2, ambas as cinzas (Cinza $P$ e Cinza T) possuem como principal fase cristalina o quartzo.

A análise térmica diferencial e termogravimétrica (Figura 3) apresentou um resultado compatível com a composição química realizada por FRX. O gráfico de ATD mostra um evento endotérmico em torno de $780{ }^{\circ} \mathrm{C}$ relativo a reação de decomposição do carbonato de cálcio em gás carbônico. Nesta mesma região a termogravimetria apresentou uma perda de massa de $2 \%$ correspondendo a um teor de $4,5 \%$ de carbonato de cálcio na amostra original. Na temperatura de $1200{ }^{\circ} \mathrm{C}$ ocorre outra perda de massa de $0,5 \%$ que pode ser explicada pela redução dos óxidos de ferro presentes na amostra. Da temperatura ambiente até em torno de $600{ }^{\circ} \mathrm{C}$ houve uma perda de massa contínua ligada possivelmente a oxidação de matéria orgânica e decomposição de hidróxidos e saída de água adsorvida na amostra [43].

O resultado de ATD/TG apresentou ser típico de uma amostra que passou por processo de queima, houve uma pequena perda de massa da amostra, indicando a oxidação de carbono remanescente da queima incompleta ocorrida. Não foram registrados eventos endotérmicos ou exotérmicos significativos neste ensaio.

A Figura 4 ilustra a granulometria das cinzas estudadas, onde a Cinza P apresenta partículas com diâmetro entre $0,5 \mathrm{e}$ $90 \mu \mathrm{m}$, já a Cinza T, possui partículas com diâmetro entre

0,1 e $70 \mu \mathrm{m}$, mostrando-se mais fina. A Tabela 3 apresenta os resultados de diâmetros médios de partículas em comparação com a granulometria do cimento Portland CP-IV [44].

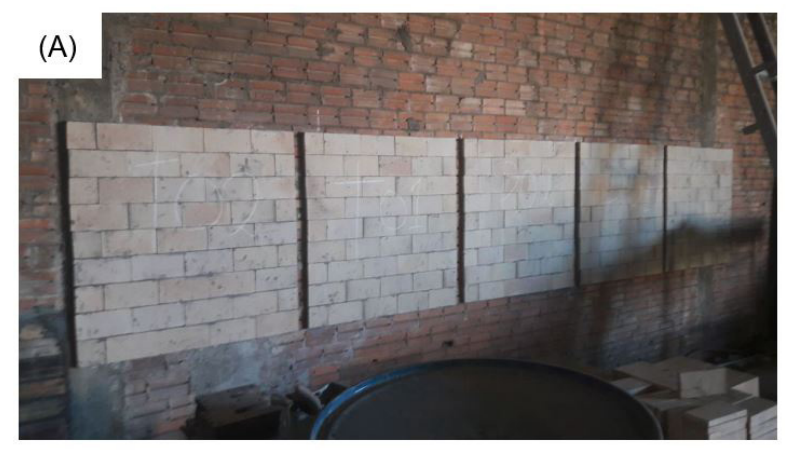

(B)
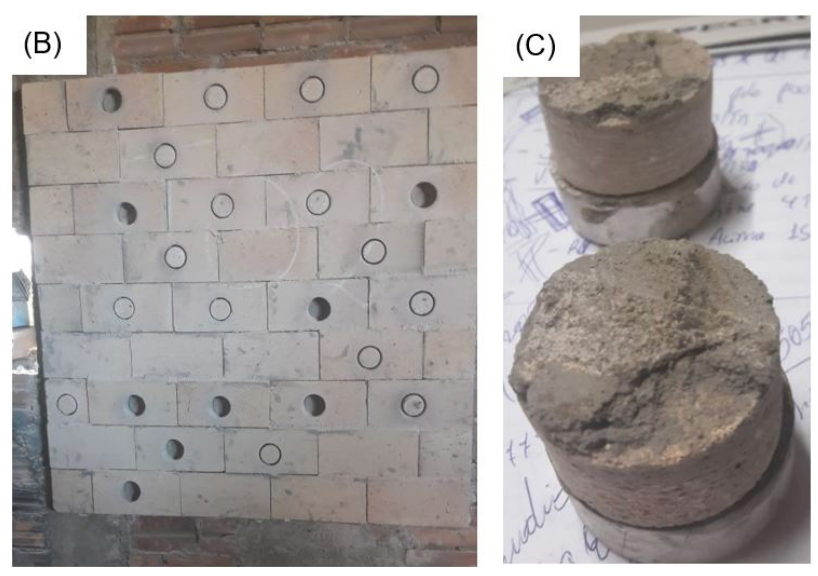

Figura 1. (A) Paredes refratárias para ensaios; (B) Parede refratária durante ensaio de arrancamento; (C) Corpos de prova após ensaio de arrancamento.

Tabela 2. Composição química da argila refratária e dos resíduos estudados

\begin{tabular}{cccc}
\hline \multirow{2}{*}{ Elementos } & \multicolumn{3}{c}{ Composição química (\% em massa) } \\
\cline { 2 - 4 } & $\begin{array}{c}\text { Argila } \\
\text { refratária }\end{array}$ & Cinza P & Cinza T \\
\hline $\mathrm{SiO}_{2}$ & 60,81 & 59,75 & 61,60 \\
$\mathrm{Al}_{2} \mathrm{O}_{3}$ & 22,11 & 23,39 & 23,10 \\
$\mathrm{Fe}_{2} \mathrm{O}_{3}$ & 1,72 & 3,18 & 5,51 \\
$\mathrm{~K}_{2} \mathrm{O}$ & 2,42 & 1,58 & 3,05 \\
$\mathrm{Na}_{2} \mathrm{O}$ & 0,38 & 0,28 & 0,66 \\
$\mathrm{CaO}$ & 0,34 & 3,93 & 1,50 \\
$\mathrm{MgO}$ & 0,47 & 0,54 & 1,25 \\
$\mathrm{MnO}$ & $<0,05$ & $<0,05$ & 0,24 \\
$\mathrm{P}_{2} \mathrm{O}_{5}$ & $<0,05$ & $<0,05$ & 0,08 \\
$\mathrm{TiO}_{2}$ & 0,88 & 1,06 & 1,17 \\
$\mathrm{ZnO}$ & $<0,1$ & 0,44 & $<0,1$ \\
$\mathrm{Perda}$ ao fogo & 9,27 & 3,31 & 1,05 \\
\hline
\end{tabular}

Tabela 3. Granulometria das Cinzas e do cimento CP-IV [44]

\begin{tabular}{lccc}
\hline & CP-IV & Cinza P & Cinza T \\
\hline Diâmetro a 10\% $(\mu \mathrm{m})$ & 5,31 & 9,92 & 2,35 \\
Diâmetro a 50\% $(\mu \mathrm{m})$ & 17,05 & 32,95 & 14,42 \\
Diâmetro a 90\% $(\mu \mathrm{m})$ & 39,58 & 56,83 & 37,25 \\
Diâmetro médio $(\mu \mathrm{m})$ & 20,18 & 33,44 & 17,66 \\
\hline
\end{tabular}




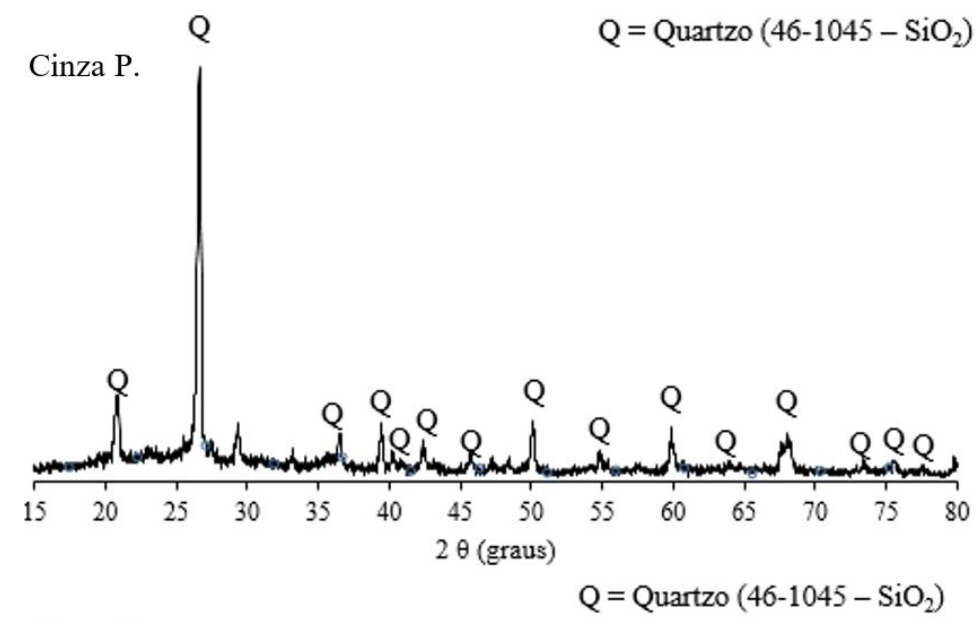

Cinza T.

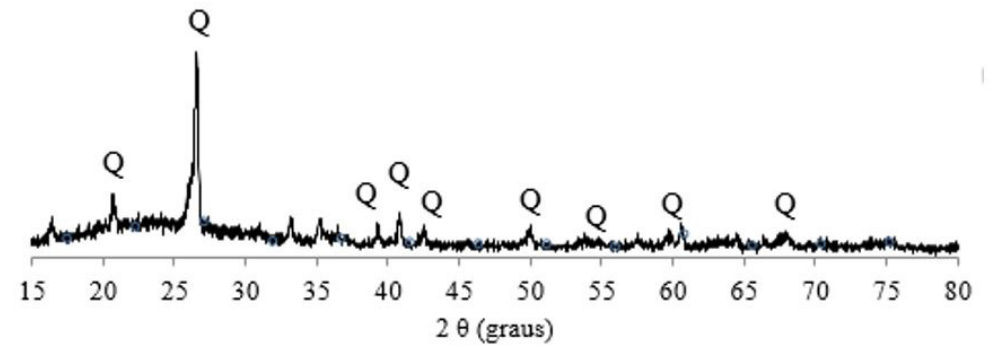

Figura 2. Difratograma de raios $\mathrm{X}$ das cinzas estudadas.
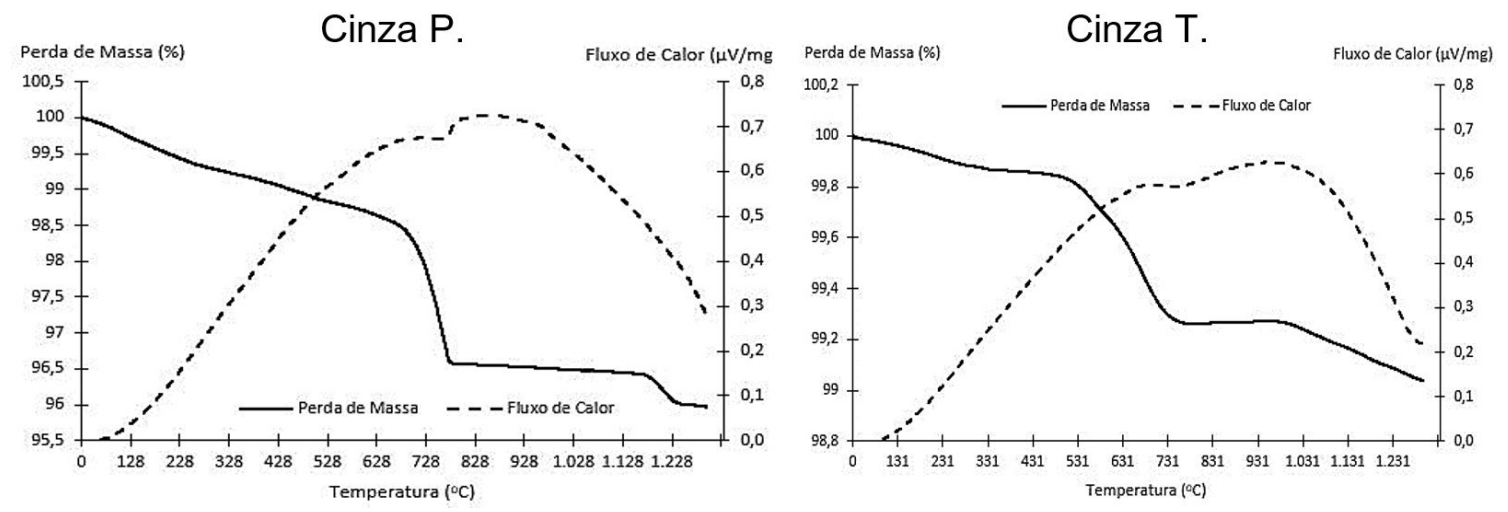

Figura 3. Análise térmica das cinzas estudadas.

A partir da Tabela 3 pode-se afirmar que, em comparação ao cimento, a Cinza $\mathrm{T}$ é mais fina, enquanto a Cinza $\mathrm{P}$ possui partículas maiores.

A Tabela 4 apresenta os resultados dos ensaios de resistência à compressão dos corpos de prova confeccionados para o estudo da atividade pozolânica à cal.

As cinzas ( $\mathrm{P} \mathrm{e} \mathrm{T}$ ) obtiveram valores para o ensaio de resistência mecânica à compressão da análise de atividade pozolânica de 4,81 e 5,41 respectivamente, sendo inferiores a exigidas na NBR 12653 [41], que determina resistência mínima à compressão necessária de $6 \mathrm{MPa}$. Desta forma estes resíduos não podem ser considerados como pozolânicos, apesar de apresentarem algumas características deste tipo de material.

Na Tabela 5 são indicados os valores de área superficial específica para cada uma das cinzas estudadas.
Tabela 4. Resistência à compressão da análise de atividade pozolânica

\begin{tabular}{ccc}
\hline \multirow{2}{*}{ Corpos de Prova } & \multicolumn{2}{c}{ Resistência à Compressão (MPa) } \\
\cline { 2 - 3 } & Cinza $\mathbf{P}$ & Cinza $\mathbf{~}$ \\
\hline CP 1 & 5,06 & 4,35 \\
CP 2 & 4,74 & 5,71 \\
CP 3 & 4,42 & 6,60 \\
CP 4 & 4,99 & 4,96 \\
Média & $\mathbf{4 , 8 1}$ & $\mathbf{5 , 4 1}$ \\
Desvio Padrão & $\mathbf{0 , 2 9}$ & $\mathbf{0 , 9 7}$ \\
\hline
\end{tabular}

Tabela 5. Área superficial específica das cinzas

\begin{tabular}{lcc}
\hline & Cinza P & Cinza T \\
\hline Área superficial específica & $6,313 \mathrm{~m}^{2} / \mathrm{g}$ & $0,558 \mathrm{~m}^{2} / \mathrm{g}$ \\
\hline
\end{tabular}


As cinzas apresentaram grande diferença em sua área superficial. Valores maiores de área superficial tornam a sinterização do material mais rápida [45]. Desta forma, a Cinza $\mathrm{P}$ tende a queimar com mais facilidade que a Cinza $\mathrm{T}$.

A Figura 5 ilustra as linhas dilatométricas à verde das formulações com a argila refratária, cinzas e cimento.

Os ensaios comparativos mostram que a adição de cinzas, não importando o tipo, aumenta significativamente a fundência. Este fato pode ser visualizado pela maior retração em altas temperaturas, acima de $1050^{\circ} \mathrm{C}$. A adição de $10 \%$ de cinza de termoelétrica a carvão apresentou a maior fundência. Para as aplicações usuais de uma churrasqueira a carvão usada comumente na construção civil em prédios residenciais, a temperatura atinge patamares menores, na casa dos $500^{\circ} \mathrm{C}$ [43], e na Figura 5 percebe-se que em nenhuma formulação atinge-se a fundência abaixo de temperaturas que são usualmente utilizadas em churrasqueiras, indicando que todas as formulações estão aptas para serem usadas nesta temperatura.

Para todas as formulações pode ser visualizada expansão característica do quartzo alfa para quartzo beta na temperatura em torno de $573^{\circ} \mathrm{C}$, compatível com os resultados de DRX destas amostras. Para a faixa de temperaturas entre a ambiente e $500^{\circ} \mathrm{C}$ acontecem as reações de desidroxilação e oxidação de matéria orgânica, por isso acontece um nível pequeno de variação das dimensões destes corpos de prova para estas temperaturas. A maior retração das formulações em temperaturas elevadas pode ser explicada pela presença de óxidos fundentes como o óxido de potássio, sódio e ferro nas cinzas o que contribui para o aumento desta característica $[11,43]$.

O objetivo de serem realizadas as dilatometrias das formulações já queimadas foi a determinação dos coeficientes de dilatação térmica médios $(\alpha)$, entre a temperatura ambiente de $25^{\circ} \mathrm{C}$ e $325^{\circ} \mathrm{C}$ que podem ser visualizados na Tabela 6 .

Os resultados indicam que houve alterações nos valores de coeficiente de dilatação térmica de acordo com adição dos resíduos em relação a dilatação do cimento puro. Com a substituição do cimento pela Cinza P, notou-se que os valores de $\alpha$ diminuíram conforme a quantidade adicionada foi aumentando. Já com a substituição do cimento pela Cinza T, os valores de $\alpha$ aumentaram. Isto indica que, com o aumento da temperatura, a argamassa das formulações P5 e P10 dilata mais que a argamassa padrão $(\mathrm{C})$, enquanto as argamassas das formulações T5 e T10 dilatam menos que a argamassa padrão, mostrando-se mais indicadas pois a tendência de aparecimento de trincas no produto final será menor.

A Figura 6 apresenta as linhas dilatométricas das amostras submetidas à queima.

Através da Figura 6 nota-se que houve diferença significativa no padrão de dilatação entre as formulações estudadas. A cinza da indústria cerâmica diminui a dilatação enquanto que a cinza da termoelétrica a carvão aumenta a dilatação. O cimento apresenta dilatação intermediária. A presença de quartzo pode ser visualizada em todas as formulações na expansão em torno de $573{ }^{\circ} \mathrm{C}$, sendo que a adição de Cinza $\mathrm{P}$ incrementou este
Cinza P.

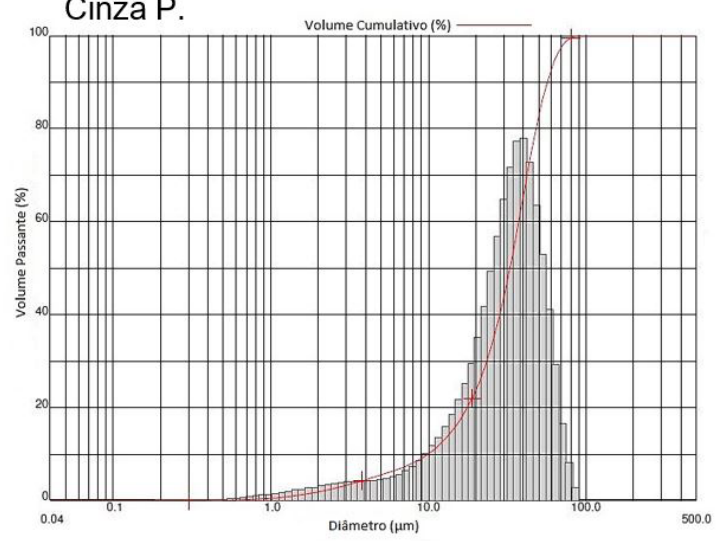

Cinza T.

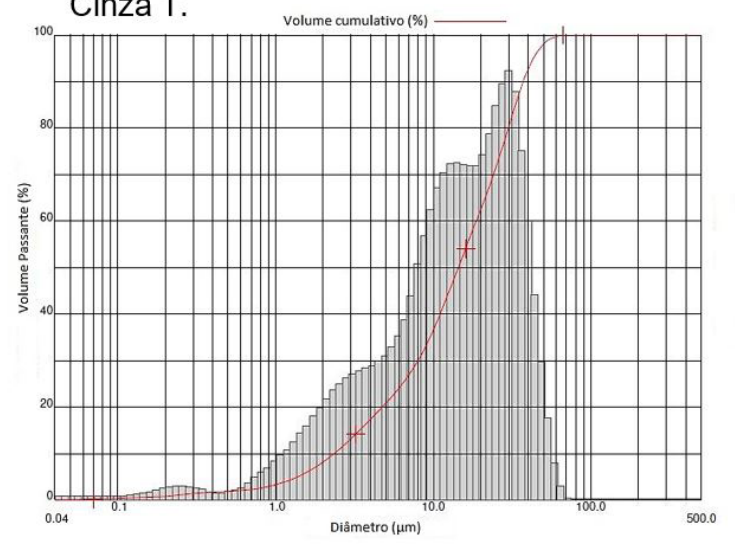

Figura 4. Granulometria das cinzas estudadas.

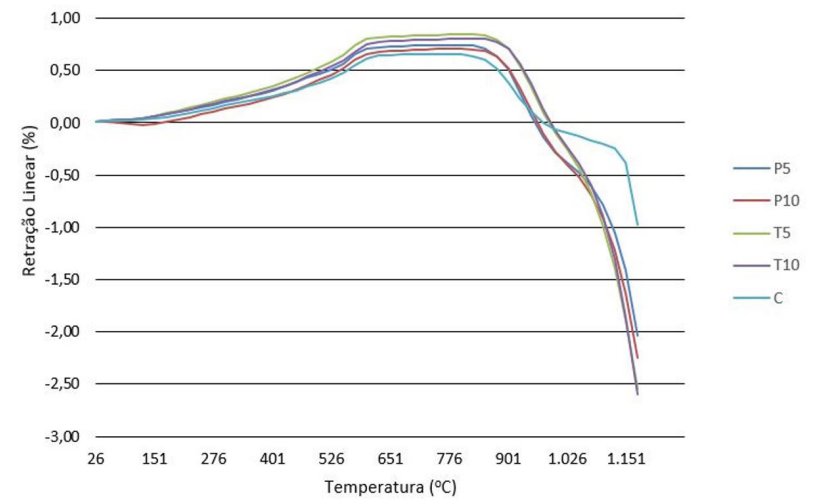

Figura 5. Dilatometria das formulações à verde.

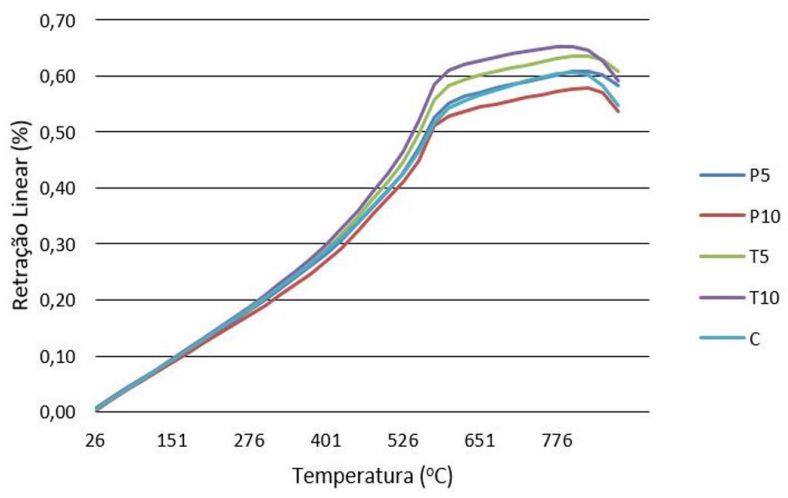

Figura 6. Dilatometria das formulações sinterizadas. 
Tabela 6. Coeficiente de dilatação térmica e temperatura de amolecimento das formulações

\begin{tabular}{ccc}
\hline Formulação & $\boldsymbol{\alpha}\left({ }^{\circ} \mathbf{C}-\mathbf{1}\right)$ & Amolecimento \\
\hline P5 & $7,18 \times 10-6$ & $819,2{ }^{\circ} \mathrm{C}$ \\
P10 & $6,83 \times 10-6$ & $818,5^{\circ} \mathrm{C}$ \\
T5 & $7,32 \times 10-6$ & $817,5^{\circ} \mathrm{C}$ \\
T10 & $7,50 \times 10-6$ & $791,0{ }^{\circ} \mathrm{C}$ \\
C & $7,28 \times 10-6$ & $799,0{ }^{\circ} \mathrm{C}$ \\
\hline
\end{tabular}

Tabela 7. Resistência ao arrancamento das formulações

\begin{tabular}{cccccc}
\hline \multirow{2}{*}{ CP } & \multicolumn{5}{c}{ Resistência ao arrancamento (MPa) } \\
\cline { 2 - 6 } & $\mathbf{P 5}$ & $\mathbf{P 1 0}$ & $\mathbf{T 5}$ & $\mathbf{T 1 0}$ & $\mathbf{C}$ \\
\hline 1 & 0,064 & 0,083 & 0,121 & 0,069 & 0,144 \\
2 & 0,049 & 0,112 & 0,067 & 0,043 & 0,110 \\
3 & 0,087 & 0,063 & 0,114 & 0,083 & 0,110 \\
4 & 0,135 & 0,050 & 0,075 & 0,060 & 0,161 \\
5 & 0,168 & 0,094 & 0,071 & 0,057 & 0,169 \\
6 & 0,077 & 0,057 & 0,075 & 0,101 & 0,087 \\
7 & 0,084 & 0,096 & 0,101 & 0,081 & 0,168 \\
8 & 0,057 & 0,081 & 0,109 & 0,114 & 0,073 \\
9 & 0,080 & 0,072 & 0,100 & 0,062 & 0,171 \\
10 & 0,101 & 0,076 & 0,116 & 0,098 & 0,137 \\
11 & 0,112 & 0,073 & 0,096 & 0,062 & 0,026 \\
12 & 0,093 & 0,061 & 0,091 & 0,093 & 0,145 \\
Média & $\mathbf{0 , 0 9 2}$ & $\mathbf{0 , 0 7 7}$ & $\mathbf{0 , 0 9 5}$ & $\mathbf{0 , 0 7 7}$ & $\mathbf{0 , 1 2 5}$ \\
Desvio Padrão & $\mathbf{0 , 0 3 4}$ & $\mathbf{0 , 0 1 8}$ & $\mathbf{0 , 0 1 9}$ & $\mathbf{0 , 0 2 2}$ & $\mathbf{0 , 0 4 5}$ \\
\hline
\end{tabular}

evento, nesta temperatura. Com relação a temperatura máxima, até se atingir o amolecimento, as temperaturas mantiveram-se semelhantes ao padrão das amostras a verde.

A Tabela 7 apresenta os resultados de resistência de aderência à tração (arrancamento) para as formulações.

A partir dos resultados do ensaio de resistência ao arrancamento notou-se que, em comparação com a argamassa padrão, todas as formulações apresentaram diminuição na sua resistência ao arrancamento. A redução da resistência mostrou-se gradativa conforme a quantidade de cimento substituída pelas cinzas foi aumentando.

No caso da Cinza $P$, a resistência diminuiu em $26,4 \%$ com a substituição de 5\% do cimento e em $38,4 \%$ com a substituição de $10 \%$. Já para a Cinza T, as substituições de
5 e $10 \%$ do cimento resultaram em uma diminuição de $24 \%$ e $38,4 \%$ respectivamente.

Apesar destas reduções, todas as formulações apresentaram resistência suficiente e podem ser utilizadas pois se enquadram na classe A1 da NBR 13281 [46], com resistência menor que $0,2 \mathrm{MPa}$.

\section{Conclusão}

Após o estudo da substituição do cimento em argamassas refratárias por cinzas de indústria cerâmica e cinzas de termoelétrica de carvão, puderam ser feitas algumas afirmações sobre as suas propriedades.

As análises químicas e físicas dos resíduos confirmaram que, apesar de possuírem algumas características de materiais pozolânicos, como a presença em grandes quantidades dos óxidos de silício, alumínio e ferro e uma baixa perda ao fogo. Em termos de resistência à compressão, estes resíduos não podem ser denominados como pozolânicos por não alcançarem a resistência mínima de $6 \mathrm{MPa}$.

Conforme esperava-se as análises térmicas feitas nas cinzas apresentaram resultados compatíveis com as análises químicas feitas por FRX. Para a análise granulométrica, em comparação ao cimento, a Cinza P apresentou partículas maiores enquanto a Cinza T apresentou partículas mais finas. Já em relação à área superficial, a Cinza P apresentou maiores valores que a Cinza $\mathrm{T}$, indicando que a sua queima ocorre de forma mais rápida.

Com estes resultados, e após definidas as formulações, pode-se concluir, através da análise dilatométrica que todas as formulações podem ser aplicadas na construção civil pois possuem temperatura fundente maiores que $500{ }^{\circ} \mathrm{C}$, temperatura máxima que uma churrasqueira comercial pode chegar. A Cinza T mostrou-se mais vantajosa, pois apresentou menor coeficiente de dilatação linear em relação à argamassa padrão sem adição de resíduos, indicando a ocorrência de menos trincas conforme o aumento da quantidade adicionada.

Já quanto à resistência de aderência à tração, todas as formulações apresentaram diminuição nos valores em comparação com a argamassa C (padrão). Este fator, porém, não influi na aplicabilidade das argamassas por todas as formulações apresentaram resistências apropriadas.

\section{Referências}

1 Sindicato Nacional da Industria de Cimento - SNIC. Dados do setor. 2017 [acesso em 25 Jan. 2018]. Disponível em: snic.org.br/numeros-do-setor.php

2 Inocente JM, Peterson M, Elyseu F, Nandi VS, Zaccaron A, Silva MO, et al. Utilização de DSC para verificação da pozolanicidade de metacaulim ativado em reator flash. In: Associação Brasileira de Cerâmica. Anais do $63^{\circ}$ Congresso Brasileiro de Cerâmica; 2019; Bonito, MS. São Paulo: ABCeram; 2019. 708 p.

3 Foletto EL, Hoffmann R, Hoffmann RS, Portugal UL Jr, Jahn SL. Aplicabilidade das cinzas da casca de arroz. Quimica Nova. 2005;28(6):1055-1060. http://dx.doi.org/10.1590/S0100-40422005000600021.

4 Zaccaron A, Fernandes P, Nandi VS, Rosso F. Incorporação de escória de alto forno a carvão vegetal em massa de cerâmica vermelha. Cerâmica Industrial. 2020;25(1):34-42. http://dx.doi.org/10.4322/cerind.2019.018. 
Desenvolvimento de uma formulação de argamassa refratária com utilização de resíduos industriais para aplicação na construção civil

5 Faria KCP, Gurgel RF, Holanda JNF. Recycling of sugarcane bagasse ash waste in the production of clay bricks. Journal of Environmental Management. 2012;101:7-12. http://dx.doi.org/10.1016/j.jenvman.2012.01.032.

6 Cordeiro GC, Toledo RD Fo, Fairbairn EMR. Caracterização de cinzas do bagaço de cana-de-açucar para emprego como pozolana em materiais cimentícios. Química Nova. 2009;32(1):82-86. http://dx.doi.org/10.1590/S010040422009000100016.

7 Xu Q, Ji T, Gao SJ, Yang Z, Wu N. Characteristics and applications of sugar cane bagasse ash waste in cementitious materials. Materials. 2018;12(1):1-19. http://dx.doi.org/10.3390/ma12010039.

8 Pode R. Potential applications of rice husk ash waste from rice husk biomass power plant. Renewable \& Sustainable Energy Reviews. 2016;53:1468-1485. http://dx.doi.org/10.1016/j.rser.2015.09.051.

9 Rodríguez de Sensale G. Strength development of concrete with rice-husk ash. Cement and Concrete Composites. 2006;28(2):158-160. http://dx.doi.org/10.1016/j.cemconcomp.2005.09.005.

10 Bezerra IMT, Souza J, Carvalho JQB, Neves GA. Aplicação da cinza da casca do arroz em argamassas de assentamento. Revista Brasileira de Engenharia Agrícola e Ambiental. 2011;15(6):639-645. http://dx.doi. org/10.1590/S1415-43662011000600015.

11 Borlini MC, Sales HF, Vieira CMF, Conte RA, Pinatti DG, Monteiro SN. Cinza da lenha para aplicação em cerâmica vermelha Parte I: características da cinza. Cerâmica. 2005;51(319):192-196.

12 Pereira SI, Peterson M, Zaccaron A, Nandi VS, Fernandes P. Incorporação da cinza do eucalipto em massa de cerâmica vermelha. Revista Eletrônica Mater. e Process. 2016;11:68-72.

13 Borges D, Bianchi DVG, Akasaki JL, Trentin TFS. Uso de cinza de madeira de eucalipto em compostos cimentícios, uma alternativa sustentável. Periódico Técnico e Científico Cidades Verdes. 2017;5:89-103.

14 Sampaolo A, Relini G. Coal ash for artificial habitats in Italy. Bulletin of Marine Science. 1994;55:1277-1294. http://dx.doi.org/10.1007/978-94-011-4215-1_21.

15 Sena da Fonseca B, Galhano C, Seixas D. Technical feasibility of reusing coal combustion by-products from a thermoelectric power plant in the manufacture of fi red clay bricks. Applied Clay Science. 2015;104:189-195. http:// dx.doi.org/10.1016/j.clay.2014.11.030.

16 Zanin TL, Klitzke W, Luz LFL Jr. Estudo da influência da adição de cinzas de carvão mineral nas propriedades da cerâmica vermelha. Cerâmica. 2013;59(350):231-234. http://dx.doi.org/10.1590/S0366-69132013000200006.

17 Adesanya DA, Raheem AA. Development of corn cob ash blended cement. Construction \& Building Materials. 2009;23(1):347-352. http://dx.doi.org/10.1016/j.conbuildmat.2007.11.013.

18 Rodier L, Bilba K, Onésippe C, Arsène MA. Study of pozzolanic activity of bamboo stem ashes for use as partial replacement of cement. Mater. Struct. Constr. 2017;50(1):1-14. http://dx.doi.org/10.1617/s11527-016-0958-6.

19 Zhou S, Zhang X, Chen X. Pozzolanic activity of feedlot biomass (cattle manure) ash. Construction \& Building Materials. 2012;28(1):493-498. http://dx.doi.org/10.1016/j.conbuildmat.2011.09.003.

20 Fava G, Ruello ML, Corinaldesi V. Paper mill sludge ash as supplementary cementitious material. Journal of Materials in Civil Engineering. 2011;23(6):772-776. http://dx.doi.org/10.1061/(ASCE)MT.1943-5533.0000218.

21 Nakanishi EY, Frías M, Santos SF, Rodrigues MS, Vigil de La Villa R, Rodriguez O, et al. Investigating the possible usage of elephant grass ash to manufacture the eco-friendly binary cements. Journal of Cleaner Production. 2016;116:236-243. http://dx.doi.org/10.1016/j.jclepro.2015.12.113.

22 Rossi DP, Monteiro SN, Vieira CMF. Utilização de cinza de incineração de lenha e serragem em cerâmica vermelha. In: Associação Brasieira de Cerâmica. Anais do 55 Congresso Brasileiro de Cerâmica; 2011; Porto de Galinhas, PE. São Paulo: ABCeram; 2011. p. 783-794.

23 Medeiros ENM, Sposto RM, Neves GA, Menezes RR. Incorporação de cinza de lenha, lodo de estação de tratamento de água e cinza de casca de arroz em massa cerâmica: utilização da técnica de planejamento. Cerâmica. 2010;56(340):399-404. http://dx.doi.org/10.1590/S0366-69132010000400014.

24 Mendonça SF, Tenório TM, Marques SKJ. Estudo da incorporação de cinzas do bagaço de cana- de-açúcar em formulações para fabricação de tijolos solo-cimento. In: Rede Norte Nordeste de Educação Profissional e Tecnológica. Anais do VII Congresso de Pesquisa e Inovação da Rede Norte Nordeste de Educação Tecnológica; 2012; Palmas, TO. Alagoas: CONNEPI; 2012. p. 1-7.

25 Valenciano MDCM, Freire WJ. Características físicas e mecânicas de misturas de solo, cimento e cinzas de bagaço de cana-de-açúcar. Eng. Agrícola. 2004;24(3):484-492. http://dx.doi.org/10.1590/S0100-69162004000300001. 
26 Torkaman J, Ashori A, Sadr Momtazi A. Using wood fiber waste, rice husk ash, and limestone powder waste as cement replacement materials for lightweight concrete blocks. Construction \& Building Materials. 2014;50:432-436. http://dx.doi.org/10.1016/j.conbuildmat.2013.09.044.

27 Lopes JP, Altoé SPS, Rudnick T, Martins CH, Sales A. Utilização da cinza pesada da cana-de-açúcar para confecção de artefatos de cimento para pavimentação. In: Conselho Federal de Engenharia e Agronomia. Anais do Congresso Técnico Científico da Engenharia e da Agronomia; 2016; Foz do Iguaçu, PR. Brasília: CONTECC; 2016. p. 1-4.

28 Castro TR, Martins CH. Avaliação da adição de cinzas do bagaço de cana-de-açúcar em argamassas mistas. Ambiente Construído. 2016;16(3):137-151. http://dx.doi.org/10.1590/s1678-86212016000300097.

29 Shaikh FUA, Supit SWM, Sarker PK. A study on the effect of nano silica on compressive strength of high volume fly ash mortars and concretes. Materials \& Design. 2014;60:433-442. http://dx.doi.org/10.1016/j.matdes.2014.04.025.

30 Mohseni E, Naseri F, Amjadi R, Khotbehsara MM, Ranjbar MM. Microstructure and durability properties of cement mortars containing nano- $\mathrm{TiO}_{2}$ and rice husk ash. Construction \& Building Materials. 2016;114:656-664. http:// dx.doi.org/10.1016/j.conbuildmat.2016.03.136.

31 Siqueira JS, Souza CAG, Souza JAS. Reaproveitamento de cinzas de carvão mineral na formulação de argamassas. Cerâmica. 2012;58(346):275-279.

32 Peterson M, Nandi VS, Zaccaron A, Pereira SI, Fernandes P. Estudo da incorporação de cinza proveniente da queima de lenha de eucalipto na formulação de massa de uma industria cerâmica estrutural do sul-catarinense. Revista da UNIFEBE. 2015;1:9-18.

33 Casagrande GS, Zaccaron A, Galatto SL, Redivo RV, Rosso F, Peterson M. Estudo da incorporação de resíduos provenientes de termoelétrica e beneficiamento do carvão mineral em massa para produção de adoquin cerâmico. In: Universidade do Extremo Sul Catarinense. Anais da VIII Semana de Ciência e Tecnologia; 2017; Criciúma, SC. Criciúma: UNESC; 2017. p. 5.

34 Carvalho MA Jr. Avaliação da aderência dos revestimentos argamassados: uma contribuição à identificação do sistema de aderência mecânico [tese]. Belo Horizonte: Universidade Federal de Minas Gerais; 2005.

35 Cincotto MA. Patologia das argamassas de revestimento: análise e recomendações. 2. ed. São Paulo: IPT; 1989.

36 Sabbatini FH. Argamassas de assentamento para paredes de alvenaria resistente. 2. ed. São Paulo: ABCP; 1998.

37 Picchi FA, Agopyan V. Sistemas de qualidade: uso em empresas de construção de edifícios [tese] São Paulo: Escola Politécnica, Universidade de São Paulo; 1993.

38 Associação Brasileira de Normas Técnicas - ABNT. NBR 5751: materiais pozolânicos: determinação da atividade pozolânica com cal aos sete dias. Rio de Janeiro: ABNT; 2015.

39 Associação Brasileira de Normas Técnicas - ABNT. NBR 5739: concreto: ensaio de compressão de corpos-de-prova cilíndricos. Rio de Janeiro: ABNT; 2007.

40 Associação Brasileira de Normas Técnicas - ABNT. NBR 13528: revestimento de paredes de argamassas inorgânicas: determinação da resistência à tração. Rio de Janeiro: ABNT; 2010.

41 Associação Brasileira de Normas Técnicas - ABNT. NBR 12653: materiais pozolânicos: requisitos. Rio de Janeiro: ABNT; 2014.

42 Benvindo da Luz A, Lins FAF. Rochas \& minerais industriais: usos e especificações. 2. ed. Rio de Janeiro: CETEM/ MCT; 2008.

43 Santos LL, Soares JE Fo, Feitosa AO, Dutra RPS, Nascimento RM. Viabilidade de incorporação de cinzas da lenha de algaroba em uma massa cerâmica em função de parâmetros de processamento. In: Universidade Estadual Paulista. Anais do $21^{\circ}$ Congresso Brasileiro de Engenharia e Ciência dos Materiais; 2014; Cuiabá. Cuiabá: CBECiMat; 2014. p. 419-430.

44 Oliveira W, Oliveira H. Análise de cimento Portland CP-IV. ACIL Weber; 2015 [acesso em 15 mar. 2018 ]. Disponível em: laboratoriodeaplicacoes.blogspot.com/2015/05/analise-de-cimento-portland-cp-iv.html

45 Azevedo AGS, Strecker K, Gorgulho HF. Efeito da temperatura em processos de sinterização de pós de hidroxiapatita. Cerâmica. 2015;61(357):52-59. http://dx.doi.org/10.1590/0366-69132015613571879.

46 Associação Brasileira de Normas Técnicas - ABNT. NBR 13281: argamassa para assentamento e revestimento de paredes e tetos: requisitos. Rio de Janeiro: ABNT; 2005.

Recebido em: 7 Nov. 2019

Aceito em: 15 Maio 2020 